\title{
Study Conditions of Center of Gravity for Trivial Solution to Semi Linear Differantial Equation of Third Order in The One Critical Cases
}

Thair Younis Thanoon

thairthanoon@uomosul.edu.iq

College of Computer Sciences and Mathematics

Zena Talal Yaseen

zena-talal@uomosul.edu.iq

\section{Received on: 14/02/2011} University of Mosul, Mosul, Iraq

\section{ABSTRACT}

Accepted on: 16/05/2011

In this paper we study the conditions under which the zero solution is center of Gravity in the semi-liner case for certain third order differential equation of the form: $Y^{\prime \prime \prime}+P_{1}(t) Y^{\prime \prime}+P_{2}(t) Y^{\prime}+P_{3}(t) Y=h(t, \bar{Y})+g(t)$,

\section{We have:}

$P_{i}=\psi^{i}\left[q_{i}+w_{i}(t)\right], \quad q_{i} \in C, \quad w_{i}(t): \Delta \rightarrow C, \quad w_{i}(t)=0(1), \quad i=1,2,3$ $t \in \Delta=[a, \infty), \quad a \in N, \quad g: \Delta \rightarrow R, \bar{Y}=\left(Y, Y^{s}, Y^{\prime \prime}\right)$

The characteristic equation of the above differential equation has complex roots of the form :

$$
M_{1}=-M_{2}=i M_{0}, \quad M_{0}>0,
$$

and the other root has the following property $\operatorname{Re} M_{3}<0$.

Keywords: Stability theory ,Critical cases, Center of gravity

$$
\text { دراسة شروط مركز جاذبية الحل الصفري لمعادلة تفاضلية شبه خطية من المرتبة الثالثة في أحلى الحالات الحرجة }
$$

زينة طلال ياسين

ثائر يونس ذنون

$$
\text { كلية علوم الحاسوب والرياضيات، جامعة الموصل فئل }
$$

تاريخ قبول البحث: 2011/05/16

تاريخ استلام البحث: 2011/02/14

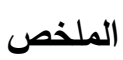

في هذا البحث سندرس شروط مركز جاذبية الحل الصفري في الحالة شبه الخطية لمعادلة تفاضلية من المرتبة

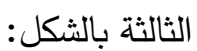

$$
Y^{n \prime}+P_{1}(t) Y^{n}+P_{2}(t) Y^{\prime}+P_{3}(t) Y=h(t, \bar{Y})+g(t)
$$

$P_{i}=\psi^{i}\left[q_{i}+w_{i}(t)\right], \quad q_{i} \in C, \quad w_{i}(t): \Delta \rightarrow C, \quad w_{i}(t)=0(1), \quad i=1,2,3$

$t \in \Delta=[a, \infty), \quad a \in N, \quad g: \Delta \rightarrow \boldsymbol{R} \quad, \bar{Y}=\left(Y, Y^{\prime}, Y^{n}\right)$

إن المعادلة المميزة للمعادلة التفاضلية أعلاه لها زوج من الجذور المعقدة بالثكل:

$M_{1}=-M_{2}=i M_{0}, \quad M_{0}>0$

والجذر الأخر يحقق الخاصية Re 0 R 0.

الكلمات المفتاحية : نظرية الاستقرار , الحالات الحرجة , مركز الجاذبية

1- المقدمة

الاستقرار مفهوم ذو أهمية متزايدة في العلوم الحديثة فهو يستخدم في الكثير من التطبيقات العلمية

$$
\text { والتكنولوجية والطبية والعسكرية وعلم الفلك وعلوم أخرى كثيرة. }
$$


وقد اقترح مفهوم الاستقرار أولاً في الميكانيك والفيزياء أثناء دراسة توازن الأجسام الواقعة تحت تأثير خارجي، فقد عرف بان الأجسام التي تمثلها مجموعة (منظومة) من المعادلات الرياضية تكون بوضع مستقر

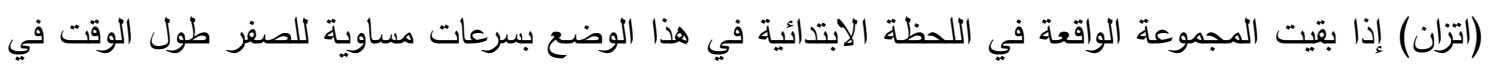

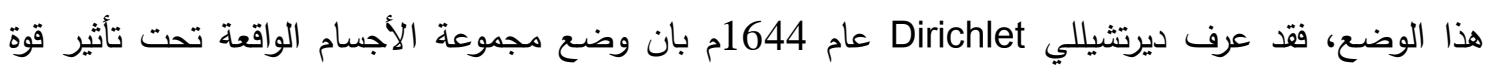

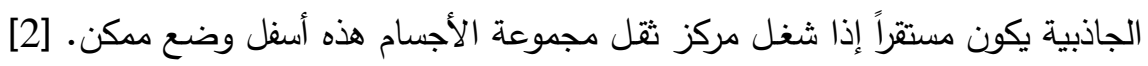

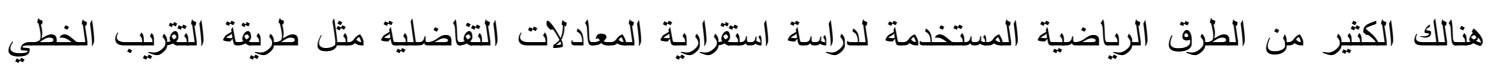

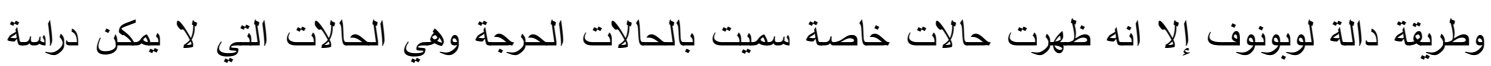
استقراريتها بالتقريب الخطي والتي يكون فيها على الأقل جذر واحد من جذور المعادلة المميزة يملك جزءاً حقيقياً

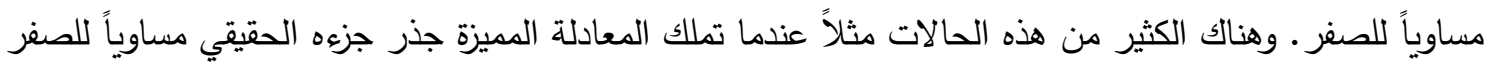
وباقي الجذور تملك جزء حقيقي سالب أو عندما يكون للمعادلة المميزة زوج أو ثلاثة جذور معقدة جزءها ها الحقيقي مساوياً للصفر وباقي الجذور تملك جزء حقيقي سالب [3] ,8] وعلى سبيل المثال نذكر الدراسات التالية : ففي [10] تم دراسة شروط استقرارية الحل الصفري لمعادلة تفاضلية من الرتبة-n في الحالة شبه الخطية والتي تمتلك الثكل :

$$
Y^{(n)}+P_{1}(t) Y^{(n-1)}+\cdots+P_{n}(t) Y=\varphi\left[t, Y, Y^{\prime}, \ldots, Y^{(n-1)}\right]
$$

في إحدى الحالات الحرجة عندما المعادلة المميزة لها تملك جذراً بالثكل :

$$
M_{0}>0, t \rightarrow \infty \text { b ع } M_{1}=i M_{0}
$$

وباقي جذور المعادلة تحقق الخاصية

Re $M_{K}<-E, \quad E>0, \quad K=2,3, \ldots, n$

أما في [11] فقد تم إيجاد الشروط التي يكون فيها الحل الصفري مركزاً للجاذبية والشروط التي لا يكون فيها مركزاً للجاذبية لمعادلة تفاضلية من المرتبة النونية ذات معاملات متغيرة ببطئ في الحالة شبه الخطية وتملك فلك فئي الثكل : $X^{(n)}+P_{1}(\sigma t) X^{(n-1)}+\cdots+P_{n}(\sigma t) X=h\left(\sigma t, X, X^{\prime}, X^{n}, \ldots, X^{(n-1)}\right)+g(\sigma t)$ في إحدى الحالات الحرجة عندما المعادلة المميزة لها تملك جذراً بالثكل : : وباقي جذور المعادلة تحقق الخاصية :

Re $\lambda_{K}<-M, \quad M>0, \quad K=2,3, \ldots, n$

أما في [9] فقد تم دراسة شروط وجود منحني جاذبية الحل لمعادلة تفاضلية غير ذاتية وشبه خطية من الرتبة الثانية عندما تحتوي معاملاتها والحد المطلق فيها على ه وتملك دود الثكل : $Y^{\prime \prime}+\lambda(\sigma t) Y=g(\sigma t)+F\left(\sigma t, Y, Y^{\prime}\right), \quad 0<\sigma<1$ إن مفهوم مركز الجاذبية center of gravity غالباً ما يستخدم في الفيزياء عندما تتجذب الحركة بتأثير قوة تسىى بالقوة المركزية عند نقطة ثابتة تسمى مركز الجاذبية [6] وقد استخدم مفهوم مركز الجاذبية في

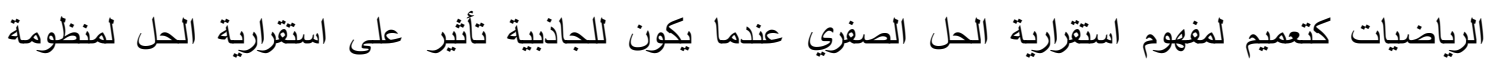
المعادلات حيث ستتجذب جميع الحلول للمعادلات التفاضلية نحو الدحور المحاذي. 
[1] تعريف (1)

يسمى الحل الصفري . $i=1, \ldots, n$ ، بؤدي إلى أن ع $\left|X_{i}\left(t_{0}\right)\right|<\delta=\delta\left(\varepsilon, t_{0}\right)$

[1] تعريف (2) إذا تحقق التعريف (1) وكان (1) =0 = (2) جاذبية محاذي 3- تحويلات مساعدة لكي يتم إيجاد شروط مركز جاذبية الحل الصفري للمعادلة التفاضلية (1) سوف نستخدم المأخوذات التالية : [4] مأخوذة (1) إن التحويل $\left.\begin{array}{l}y=\psi X_{1} \\ y^{\prime}=\psi^{2} X_{2} \\ y^{\prime \prime}=\psi^{3} X_{3}\end{array}\right\}$

وبدوره سيحول المعادلة التفاضلية (1) إلى نظام من المعادلات التفاضلية وكما يلي : $X_{1}^{\prime}=-\psi^{-1} \psi^{\prime} X_{1}+\psi X_{2}$ $X_{2}^{\prime}=-2 \psi^{-1} \psi^{\prime} X_{2}+\psi \quad X_{3}$

$X_{3}^{\prime}=-P_{3}(t) \psi^{-2} X_{1}-P_{2}(t) \psi^{-1} X_{2}-\left(P_{1}(t)+3 \psi^{-1} \psi^{\prime}\right) X_{3}+F_{1}(t, X)+\psi^{-3} g(t)$

حيث أن $\left|F_{1}(t, X)\right| \leq L\left(X_{1}+\psi X_{2}+\psi^{2} X_{3}\right)^{1+\beta} \quad, L=L^{*} \psi^{\beta-2}$

[4] مأخوذة (2) التحويل $M=-C X$ $C=\left[\begin{array}{ccc}\left(i \mu_{\mathrm{o}}\right)^{2}+q_{1}\left(i \mu_{\mathrm{o}}\right)+q_{2} & i \mu_{\mathrm{o}}+q_{1} & 1 \\ \left(-i \mu_{\mathrm{o}}\right)^{2}+q_{1}\left(i \mu_{\mathrm{o}}\right)+q_{2} & -i \mu_{\mathrm{o}}+q_{1} & 1 \\ 1 & 0 & 0\end{array}\right]$ حيث أن سيحول النظام التفاضلي (3) إلى النظام بالثكل 


$$
\begin{aligned}
& M_{1}^{\prime}=\psi\left[i \mu_{\mathrm{o}} M_{1}-a_{1} M_{3}\right]+F_{2}+\psi^{-3} g(t) \\
& M_{2}^{\prime}=\psi\left[q_{1} M_{1}+\left(-i \mu_{\mathrm{o}}-q_{1}\right) M_{2}+a_{2} M_{3}\right]+F_{2}+\psi^{-3} g(t) \\
& M_{3}^{\prime}=\psi\left[\frac{1}{2 i \mu_{\circ}} M_{1}-\frac{1}{2 i \mu_{\circ}} M_{2}\right] \\
& a_{1}=q_{3}+\left(i \mu_{\mathrm{o}}\right)^{3}+\left(i \mu_{\mathrm{o}}\right) q_{2}+\left(i \mu_{\mathrm{o}}\right)^{2} q_{1} \\
& a_{2}=-q_{3}+\left(i \mu_{\mathrm{o}}\right)^{3}+\left(i \mu_{\mathrm{o}}\right) q_{2}+\left(i \mu_{\mathrm{o}}\right)^{2} q_{1} \\
& \left|F_{2}\right| \leq L\left[\frac { 1 } { - 2 i \mu _ { \mathrm { o } } } \left[\left(-\psi+\psi^{2}\left(-i \mu_{\mathrm{o}}+q_{1}\right)\right) M_{1}+\left(\psi-\psi^{2}\left(i \mu_{\mathrm{o}}+q_{1}\right)\right) M_{2}+\right.\right. \\
& \left.\left.+\left(-2 i \mu_{\mathrm{o}}-\psi^{2}\left(-2 q_{1}\left(i \mu_{\mathrm{o}}\right)^{2}-2\left(i \mu_{\mathrm{o}}\right)^{3}-2 i \mu_{\mathrm{o}} q_{2}\right)\right) M_{3}\right]\right]^{1+\beta}
\end{aligned}
$$

\section{مأخوذة (3) [4]}

باستخدام التحويل التالي

$$
\left.\begin{array}{l}
M_{1}=y_{1} \\
M_{2}=y_{2} \\
M_{3}=k y_{1}+\bar{k} y_{2}+y_{3} \quad, \quad k, \bar{k} \in C
\end{array}\right\}
$$

وباستخدام النظام (5) ممكن كتابة النظام السابق كالآتي:

$$
\begin{aligned}
& y_{1}^{\prime}=\psi\left(d_{2} y_{1}-a_{1} \bar{k} y_{2}+-a_{1} y_{3}\right)+F_{3}+\psi^{-3} g(t) \\
& y_{2}^{\prime}=\psi\left(d_{1} y_{1}-d_{3} y_{2}+a_{2} y_{3}\right)+F_{3}+\psi^{-3} g(t) \\
& \left.y_{3}^{\prime}=\psi\left[\left(\frac{1}{2 i \mu_{\circ}}+d_{4}\right) y_{1}+\left(-\frac{1}{2 i \mu_{\circ}}+d_{5}\right) y_{2}+d_{6} y_{3}\right]-F_{3}(k+\bar{k})-\psi^{-3} g(k+\bar{k})\right] \\
& d_{1}=q_{1}+a_{2} k \quad, d_{4}=-k d_{2}-\bar{k} d_{1} \\
& d_{2}=i \mu_{\circ}-a_{1} k \quad, d_{5}=a_{1} k \bar{k}+\bar{k} d_{3} \\
& d_{3}=i \mu_{0}+q_{1}-a_{2} \bar{k} \quad, d_{6}=k a_{1}-\bar{k} a_{2} \\
& \left|F_{3}\right| \leq L\left[\frac{-\psi+\psi^{2}\left(-i \mu_{\mathrm{o}}+q_{1}\right)+k\left(-2 i \mu_{\mathrm{o}}-\psi^{2}\left(-2\left(i \mu_{\mathrm{o}}\right)^{2} q_{1}-2\left(i \mu_{\mathrm{o}}\right)^{3}-2 i \mu_{\mathrm{o}} q_{2}\right)\right)}{-2 i \mu_{\mathrm{o}}} y_{1}+\right. \\
& +\frac{\psi-\psi^{2}\left(i \mu_{\circ}+q_{1}\right)+\bar{k}\left(-2 i \mu_{\mathrm{o}}-\psi^{2}\left(-2\left(i \mu_{\mathrm{o}}\right)^{2} q_{1}-2\left(i \mu_{\mathrm{o}}\right)^{3}-2 i \mu_{\mathrm{o}} q_{2}\right)\right)}{-2 i \mu_{\mathrm{o}}} y_{2}+ \\
& \left.+\frac{-2 i \mu_{\mathrm{o}}-\psi^{2}\left(-2\left(i \mu_{\mathrm{o}}\right)^{2} q_{1}-2\left(i \mu_{\mathrm{o}}\right)^{3}-2 i \mu_{\mathrm{o}} q_{2}\right)}{-2 i \mu_{\mathrm{o}}} y_{3}\right]^{1+\beta}
\end{aligned}
$$

[4أخوذة (4) [4]

من التحويل

$$
\left.\begin{array}{l}
y_{1}=z_{1}+b z_{2}+b_{3} z_{3} \\
y_{2}=b z_{1}+z_{2}+b_{3} z_{3} \\
y_{3}=-z_{3}
\end{array}\right\}
$$


نحصل على النظام التالي :

$$
\begin{aligned}
z_{1}^{\prime}= & \frac{\psi}{1-b^{2}}\left\{\left[d_{2}+b\left(-d_{1}-a_{1} \bar{k}+b d_{3}\right)-b_{3}(b-1)\left[\left(\frac{1}{2 i \mu_{\circ}}+d_{4}\right)+b\left(\frac{-1}{2 i \mu_{\circ}}+d_{5}\right)\right]\right] z_{1}+\right. \\
& +\left[b\left(d_{2}-b d_{1}+d_{3}\right)-a_{1} \bar{k}-b_{3}(b-1)\left(b\left(\frac{1}{2 i \mu_{\circ}}+d_{4}\right)-\frac{1}{2 i \mu_{\circ}}+d_{5}\right)\right] z_{2}+ \\
& \left.+\left[b_{3}\left(d_{2}+b\left(d_{3}-d_{1}\right)+b_{3}(b-1)\left(d_{4}+d_{5}\right)-a_{1} \bar{k}\right)+a_{1}+b a_{2}+b_{3}(b-1) d_{6}\right] z_{3}\right\}+ \\
& +\frac{F_{4}}{1-b^{2}}\left(1-b+b_{3}(b-1)(k+\bar{k})\right)+\frac{\psi^{-3}}{1-b^{2}}\left(1-b+b_{3}(b-1)(k+\bar{k})\right) \\
z_{2}^{\prime}= & \frac{\psi}{1-b^{2}}\left\{\left[d_{1}+b\left(-d_{2}+b a_{1} \bar{k}-d_{3}\right)-b_{3}(b-1)\left(\frac{1}{2 i \mu_{\circ}}+d_{4}+b\left(\frac{-1}{2 i \mu_{\circ}}+d_{5}\right)\right)\right] z_{1}\right. \\
& +\left[b\left(-b d_{2}+d_{1}+a_{1} \bar{k}\right)-d_{3}-b_{3}(b-1)\left(b\left(\frac{1}{2 i \mu_{\circ}}+d_{4}\right)-\frac{1}{2 i \mu_{\circ}}+d_{5}\right)\right] z_{2} \\
& \left.+\left[b_{3}\left(d_{1}-b\left(d_{2}-a_{1} \bar{k}\right)-d_{3}-b_{3}(b-1)\left(d_{4}+d_{5}\right)\right)-b a_{1}-a_{2}+b_{3}(b-1) d_{6}\right] z_{3}\right\} \\
& +\frac{F_{4}}{1-b^{2}}\left(1-b+b_{3}(b-1)(k+\bar{k})\right)+\frac{\psi^{-3} g}{1-b^{2}}\left(1-b+b_{3}(b-1)(k+\bar{k})\right) \\
z_{3}^{\prime} & =-\psi\left\{\left[\frac{1}{2 i \mu_{\circ}}+d_{4}+b\left(-\frac{1}{2 i \mu_{\circ}}+d_{5}\right)\right] z_{1}+\right. \\
& +\left[b\left(\frac{1}{2 i \mu_{\circ}}+d_{4}\right)-\frac{1}{2 i \mu_{\circ}}+d_{5}\right] z_{2}+ \\
& \left.\left.+b_{3}\left(d_{4}+d_{5}\right)-d_{6}\right] z_{3}\right\}+F_{4}(k+\bar{k})+\psi^{-3} g(k+\bar{k}) \\
\left|F_{4}\right| & \leq L\left\{\frac { 1 } { - 2 i \mu _ { \circ } } \left[-\psi+\psi^{2}\left(-i \mu_{\circ}+q_{1}\right)+k\left(-2 i \mu_{\circ}-\psi^{2}\left(-2\left(i \mu_{\circ}\right)^{3}-2 q_{1}\left(i \mu_{\circ}\right)^{2}-2 i \mu_{\circ} q_{2}\right)\right)+\right.\right. \\
& \left.+b\left(\psi-\psi^{2}\left(i \mu_{\circ}+q_{1}\right)+\bar{k}\left(-2 i \mu_{\circ}-\psi^{2}\left(-2\left(i \mu_{\circ}\right)^{3}-2 q_{1}\left(i \mu_{\circ}\right)^{2}-2 i \mu_{\circ} q_{2}\right)\right)\right)\right] z_{1}+ \\
& +\frac{1}{-2 i \mu_{\circ}}\left[b\left(-\psi+\psi^{2}\left(-i \mu_{\circ}+q_{1}\right)+k\left(-2 i \mu_{\circ}-\psi^{2}\left(-2\left(i \mu_{\circ}\right)^{3}-2 q_{1}\left(i \mu_{\circ}\right)^{2}-2 i \mu_{\circ} q_{2}\right)\right)\right)+\right. \\
& +\left(\psi-\psi^{2}\left(i \mu_{\circ}+q_{1}\right)+\bar{k}\left(-2 i \mu_{\circ}-\psi^{2}\left(-2\left(i \mu_{\circ}\right)^{3}-2 q_{1}\left(i \mu_{\circ}\right)^{2}-2 i \mu_{\circ} q_{2}\right)\right)\right] z_{2}+ \\
& +\frac{1}{-2 i \mu_{\circ}}\left[b_{3}\left(-2 i \mu_{\circ} \psi^{2}+(k+\bar{k})\left(-2 i \mu_{\circ}-\psi^{2}\left(-2\left(i \mu_{\circ}\right)^{3}-2 q_{1}\left(i \mu_{\circ}\right)^{2}-2 i \mu_{\circ} q_{2}\right)\right)\right)+\right. \\
& \left.\left.+2 i \mu_{\circ}+\psi^{2}\left(-2\left(i \mu_{\circ}\right)^{3}-2 q_{1}\left(i \mu_{\circ}\right)^{2}-2 i \mu_{\circ} q_{2}\right)\right] z_{3}\right\}^{1+\beta} \\
&
\end{aligned}
$$

مأخوذة (5) [4]

التحويل

$$
\left.\begin{array}{l}
z_{1}=w_{1} e^{i \theta} \\
z_{2}=w_{2} e^{-i \theta} \\
z_{3}=-w_{3}
\end{array}\right\}
$$

$$
\theta \in[0,2 \pi] \text { حيث ألمان }
$$
يحول النظام (9) إلى النظام التناضلي التالي: 


$$
\begin{aligned}
& w_{1}^{\prime}=\lambda_{1} w_{1}+\lambda_{2} w_{2}+\lambda_{3} w_{3}+F_{5} \frac{e^{-i \theta}}{1-b^{2}}\left(1-b+b_{3}(b-1)(k+\bar{k})\right)+ \\
& +\psi^{-3} g \frac{e^{-i \theta}}{1-b^{2}}\left(1-b+b_{3}(b-1)(k+\bar{k})\right) \\
& w_{2}^{\prime}=\lambda_{1}^{*} w_{1}+\lambda_{2}^{*} w_{2}+\lambda_{3}^{*} w_{3}+\frac{e^{i \theta} F_{5}}{1-b^{2}}\left(1-b+b_{3}(b-1)(k+\bar{k})\right)+ \\
& +\frac{e^{i \theta} \psi^{-3} g}{1-b^{2}}\left(1-b+b_{3}(b-1)(k+\bar{k})\right) \\
& w_{3}^{\prime}=\lambda_{1}^{* *} w_{1}+\lambda_{2}^{* *} w_{2}+\lambda_{3}^{* *} w_{3}-F_{5}(k+\bar{k})-\psi^{-3} g(k+\bar{k})
\end{aligned}
$$

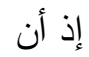

$$
\begin{aligned}
& A_{1}=d_{2}+b\left(-d_{1}-a_{1} \bar{k}+b d_{3}\right)-b_{3}(b-1)\left[\left(\frac{1}{2 i \mu_{\circ}}+d_{4}\right)+b\left(\frac{-1}{2 i \mu_{\circ}}+d_{5}\right)\right] \\
& A_{2}=b\left(d_{2}-b d_{1}+d_{3}\right)-a_{1} \bar{k}-b_{3}(b-1)\left(b\left(\frac{1}{2 i \mu_{\circ}}+d_{4}\right)-\frac{1}{2 i \mu_{\circ}}+d_{5}\right) \\
& A_{3}=b_{3}\left(d_{2}+b\left(d_{3}-d_{1}\right)-b_{3}(b-1)\left(d_{4}+d_{5}\right)-a_{1} \bar{k}\right)+a_{1}+b a_{2}+b_{3}(b-1) d_{6} \\
& \lambda_{1}=\frac{\psi}{1-b^{2}} A_{1} \quad, \quad \lambda_{2}=\frac{\psi e^{-2 i \theta}}{1-b^{2}} A_{2} \quad, \quad \lambda_{3}=\frac{-e^{-i \theta} \psi}{1-b^{2}} A_{3} \\
& B_{1}=d_{1}+b\left(-d_{2}+b a_{1} \bar{k}-d_{3}\right)-b_{3}(b-1)\left(\frac{1}{2 i \mu_{\mathrm{o}}}+d_{4}+b\left(\frac{-1}{2 i \mu_{\mathrm{o}}}+d_{5}\right)\right) \\
& B_{2}=b\left(-b d_{2}+d_{1}-a_{1} \bar{k}\right)-d_{3}-b_{3}(b-1)\left(b\left(\frac{1}{2 i \mu_{\mathrm{o}}}+d_{4}\right)-\frac{1}{2 i \mu_{\mathrm{o}}}+d_{5}\right) \\
& B_{3}=b_{3}\left(d_{1}-b\left(d_{2}-a_{1} \bar{k}\right)-d_{3}-b_{3}(b-1)\left(d_{4}+d_{5}\right)\right)-b a_{1}-a_{2}+b_{3}(b-1) d_{6} \\
& \lambda_{1}^{*}=\frac{\psi}{1-b^{2}}\left(B_{1} e^{2 i \theta}\right), \quad \lambda_{2}^{*}=\frac{\psi}{1-b^{2}} B_{2}, \quad \lambda_{3}^{*}=\frac{-\psi}{1-b^{2}} e^{i \theta} B_{3} \\
& C_{1}=\frac{1}{2 i \mu_{\mathrm{o}}}+d_{4}+b\left(-\frac{1}{2 i \mu_{\mathrm{o}}}+d_{5}\right) \\
& C_{2}=b\left(\frac{1}{2 i \mu_{\circ}}+d_{4}\right)-\frac{1}{2 i \mu_{\circ}}+d_{5} \\
& C_{3}=b_{3}\left(d_{4}+d_{5}\right)-d_{6} \\
& \lambda_{1}^{* *}=\psi\left(C_{1} e^{i \theta}\right), \quad \lambda_{2}^{* *}=\psi\left(C_{2} e^{-i \theta}\right), \quad \lambda_{3}^{* *}=-\psi C_{3}
\end{aligned}
$$




$$
\begin{aligned}
& \left|F_{5}\right| \leq L\left\{\frac { 1 } { - 2 i \mu _ { \circ } } \left[-\psi+\psi^{2}\left(-i \mu_{\circ}+q_{1}\right)+k\left(-2 i \mu_{\circ}-\psi^{2}\left(-2\left(i \mu_{\mathrm{o}}\right)^{3}-2 q_{1}\left(i \mu_{\mathrm{o}}\right)^{2}-2 i \mu_{\mathrm{o}} q_{2}\right)\right)+\right.\right. \\
& +b\left(\psi-\psi^{2}\left(i \mu_{\circ}+q_{1}\right)+\bar{k}\left(-2 i \mu_{\circ}-\psi^{2}\left(-2\left(i \mu_{\mathrm{o}}\right)^{3}-2 q_{1}\left(i \mu_{\mathrm{o}}\right)^{2}-2 i \mu_{\mathrm{o}} q_{2}\right)\right)\right] w_{1} e^{i \theta}+ \\
& +\frac{1}{-2 i \mu_{\mathrm{o}}}\left[b\left(-\psi+\psi^{2}\left(-i \mu_{\mathrm{o}}+q_{1}\right)+k\left(-2 i \mu_{\mathrm{o}}-\psi^{2}\left(-2\left(i \mu_{\mathrm{o}}\right)^{3}-2 q_{1}\left(i \mu_{\mathrm{o}}\right)^{2}-2 i \mu_{\mathrm{o}} q_{2}\right)\right)\right)+\right. \\
& +\left(\psi-\psi^{2}\left(i \mu_{\circ}+q_{1}\right)+\bar{k}\left(-2 i \mu_{\circ}-\psi^{2}\left(-2\left(i \mu_{\mathrm{o}}\right)^{3}-2 q_{1}\left(i \mu_{\mathrm{o}}\right)^{2}-2 i \mu_{\mathrm{o}} q_{2}\right)\right)\right] w_{2} e^{-i \theta}+ \\
& +\frac{1}{-2 i \mu_{\mathrm{o}}}\left[b_{3}\left(-2 i \mu_{\mathrm{o}} \psi^{2}+(k+\bar{k})\left(-2 i \mu_{\mathrm{o}}-\psi^{2}\left(-2\left(i \mu_{\mathrm{o}}\right)^{3}-2 q_{1}\left(i \mu_{\mathrm{o}}\right)^{2}-2 i \mu_{\mathrm{o}} q_{2}\right)\right)\right)+\right. \\
& \left.\left.+2 i \mu_{\mathrm{o}}+\psi^{2}\left(-2\left(i \mu_{\mathrm{o}}\right)^{3}-2 q_{1}\left(i \mu_{\mathrm{o}}\right)^{2}-2 i \mu_{\mathrm{o}} q_{2}\right)\right]\left(-w_{3}\right)\right\}^{1+\beta}
\end{aligned}
$$

[7] مأخوذة (6) لكي نقلل قيمة الدالة

$$
w_{s}=\left(\psi^{-3} g(t)\right)^{\delta} H_{s}, s=1,2,3,90<\delta<1
$$

وباستخدام النظام (11) والنظام (12) يمكننا الحصول على النظام الأتي :

$$
\left.\begin{array}{rl}
H_{1}^{\prime} & =\left[\lambda_{1}-\delta\left(\psi^{-3} g\right)^{-1}\left(\psi^{-3} g\right)^{\prime}\right] H_{1}+\lambda_{2} H_{2}+\lambda_{3} H_{3}+ \\
& +\left(\psi^{-3} g\right)^{-\delta} F_{6} \frac{e^{-i \theta}}{1-b^{2}}\left(1-b+b_{3}(b-1)(k+\bar{k})\right)+ \\
& +\left(\psi^{-3} g\right)^{1-\delta} \frac{e^{-i \theta}}{1-b^{2}}\left(1-b+b_{3}(b-1)(k+\bar{k})\right) \\
H_{2}^{\prime} & =\lambda_{1}^{*} H_{1}+\left[\lambda_{2}^{*}-\delta\left(\psi^{-3} g\right)^{-1}\left(\psi^{-3} g\right)^{\prime}\right] H_{2}+\lambda_{3}^{*} H_{3}+ \\
& +\left(\psi^{-3} g\right)^{-\delta} F_{6} \frac{e^{i \theta}}{1-b^{2}}\left(1-b+b_{3}(b-1)(k+\bar{k})\right)+ \\
& +\left(\psi^{-3} g\right)^{1-\delta} \frac{e^{i \theta}}{1-b^{2}}\left(1-b+b_{3}(b-1)(k+\bar{k})\right) \\
H_{3}^{\prime} & =\lambda_{1}^{* *} H_{1}+\lambda_{2}^{* *} H_{2}+\left[\lambda_{3}^{* *}-\delta\left(\psi^{-3} g\right)^{-1}\left(\psi^{-3} g\right)^{\prime}\right] H_{3} \\
& -\left(\psi^{-3} g\right)^{-\delta} F_{6}(k+\bar{k})-\left(\psi^{-3} g\right)^{1-\delta}(k+\bar{k})
\end{array}\right\}
$$

$$
\begin{aligned}
\left|F_{6}\right| & \leq L\left\{\frac { 1 } { - 2 i \mu _ { \circ } } \left[-\psi+\psi^{2}\left(-i \mu_{\circ}+q_{1}\right)+k\left(-2 i \mu_{\circ}-\psi^{2}\left(-2\left(i \mu_{\circ}\right)^{3}-\right.\right.\right.\right. \\
& \left.\left.-2 q_{1}\left(i \mu_{\circ}\right)^{2}-2 i \mu_{\circ} q_{2}\right)\right)+b\left(\psi-\psi^{2}\left(i \mu_{\circ}+q_{1}\right)+\right. \\
& \left.+\bar{k}\left(-2 i \mu_{\circ}-\psi^{2}\left(-2\left(i \mu_{\circ}\right)^{3}-2 q_{1}\left(i \mu_{\circ}\right)^{2}-2 i \mu_{\circ} q_{2}\right)\right)\right]\left(\psi^{-3} g\right)^{\delta} H_{1} e^{i \theta}+ \\
& +\frac{1}{-2 i \mu_{\circ}}\left[b \left(-\psi+\psi^{2}\left(-i \mu_{\circ}+q_{1}\right)+k\left(-2 i \mu_{\circ}-\psi^{2}\left(-2\left(i \mu_{\circ}\right)^{3}-\right.\right.\right.\right. \\
& \left.\left.\left.-2 q_{1}\left(i \mu_{\circ}\right)^{2}-2 i \mu_{\circ} q_{2}\right)\right)\right)+\left(\psi-\psi^{2}\left(i \mu_{\circ}+q_{1}\right)+\right. \\
& \left.+\bar{k}\left(-2 i \mu_{\circ}-\psi^{2}\left(-2\left(i \mu_{\circ}\right)^{3}-2 q_{1}\left(i \mu_{\circ}\right)^{2}-2 i \mu_{\circ} q_{2}\right)\right)\right]\left(\psi^{-3} g\right)^{\delta} H_{2} e^{-i \theta}+ \\
& +\frac{1}{-2 i \mu_{\circ}}\left[b _ { 3 } \left(-2 i \mu_{\circ} \psi^{2}+(k+\bar{k})\left(-2 i \mu_{\circ}-\psi^{2}\left(-2\left(i \mu_{\circ}\right)^{3}-\right.\right.\right.\right.
\end{aligned}
$$




$$
\begin{aligned}
& \left.\left.\left.-2 q_{1}\left(i \mu_{\mathrm{o}}\right)^{2}-2 i \mu_{\mathrm{o}} q_{2}\right)\right)\right)+2 i \mu_{\mathrm{o}}+ \\
& \left.\left.+\psi^{2}\left(-2\left(i \mu_{\mathrm{o}}\right)^{3}-2 q_{1}\left(i \mu_{\mathrm{o}}\right)^{2}-2 i \mu_{\mathrm{o}} q_{2}\right)\right]\left(-\left(\psi^{-3} g\right)^{\delta} H_{3}\right)\right\}^{1+\beta}
\end{aligned}
$$

3- النظام المساعد

نلاحظ انه من الصعب تطبيق مبدأ الاستقرار وإيجاد شروط مركز الجاذبية من النظام السابق فنلجأ إلى

$$
\text { تكوين نظام مساعد من هذا النظام الأخير وكما يأتي }
$$

نكتب النظام المساعد للنظام (13) وسنطبق على هذا النظام شروط مركز الجاذبية والذي سيملك الثكل الآتي :

$$
\begin{aligned}
& H_{1}^{\prime}=\left[\lambda_{1}-\delta\left(\psi^{-3} g\right)^{-1}\left(\psi^{-3} g\right)^{\prime}\right] H_{1}+\lambda_{2} \xi_{2}+\lambda_{3} \xi_{3}+D_{1}+D_{2} \\
& H_{2}^{\prime}=\lambda_{1}^{*} \xi_{1}+\left[\lambda_{2}^{*}-\delta\left(\psi^{-3} g\right)^{-1}\left(\psi^{-3} g\right)^{\prime}\right] H_{2}+\lambda_{3}^{*} \xi_{3}+D_{1}^{*}+D_{2}^{*} \\
& \text { حيث أن } \\
& D_{1}=\left(\psi^{-3} g\right)^{-\delta} F_{7} \frac{e^{-i \theta}}{1-b^{2}}\left(1-b+b_{3}(b-1)(k+\bar{k})\right) \\
& D_{2}=\left(\psi^{-3} g\right)^{1-\delta} \frac{e^{-i \theta}}{1-b^{2}}\left(1-b+b_{3}(b-1)(k+\bar{k})\right) \\
& D_{1}^{*}=\left(\psi^{-3} g\right)^{-\delta} F_{7} \frac{e^{i \theta}}{1-b^{2}}\left(1-b+b_{3}(b-1)(k+\bar{k})\right) \\
& D_{2}^{*}=\left(\psi^{-3} g\right)^{1-\delta} \frac{e^{i \theta}}{1-b^{2}}\left(1-b+b_{3}(b-1)(k+\bar{k})\right) \\
& D_{1}^{* *}=-\left(\psi^{-3} g\right)^{-\delta} F_{7}(k+\bar{k}) \\
& D_{2}^{* *}=-\left(\psi^{-3} g\right)^{1-\delta}(k+\bar{k})
\end{aligned}
$$$$
H_{3}^{\prime}=\lambda_{1}^{* *} \xi_{1}+\lambda_{2}^{* *} \xi_{2}+\left[\lambda_{3}^{* *}-\delta\left(\psi^{-3} g\right)^{-1}\left(\psi^{-3} g\right)^{\prime}\right] H_{3}+D_{1}^{* *}+D_{2}^{* *}
$$

$$
\left|H_{1}\right| \leq e^{\int_{T}^{t} \operatorname{Re}\left[\lambda_{1}-\delta\left(\psi^{-3} g\right)^{-1}\left(\psi^{-3} g\right)^{\prime}\right] d t}\left[|c|+\int_{T}^{t}\left(\lambda_{2} \xi_{2}+\lambda_{3} \xi_{3}+D_{1}+\right.\right.
$$$$
\left.\left.+D_{2}\right) e^{-\int_{T}^{t} \operatorname{Re}\left[\lambda_{1}-\delta\left(\psi^{-3} g\right)^{-1}\left(\psi^{-3} g\right)^{\prime}\right] d t} d t\right]
$$

$$
\begin{gathered}
\left|H_{2}\right| \leq e^{\int^{t} \operatorname{Re}\left[\lambda_{2}^{*}-\delta\left(\psi^{-3} g\right)^{-1}\left(\psi^{-3} g\right)^{\prime}\right] d t}\left[|c|+\int_{T}^{t}\left(\lambda_{1}^{*} \xi_{1}+\lambda_{3}^{*} \xi_{3}+D_{1}^{*}+\right.\right. \\
\left.\left.+D_{2}^{*}\right) e^{-\int^{t} \operatorname{Re}\left[\lambda_{2}^{*}-\delta\left(\psi^{-3} g\right)^{-1}\left(\psi^{-3} g\right)^{\prime}\right] d t} d t\right]
\end{gathered}
$$




$$
\begin{aligned}
& \left|H_{3}\right| \leq e^{\int_{T}^{t} \operatorname{Re}\left[\lambda_{3}^{* *}-\delta\left(\psi^{-3} g\right)^{-1}\left(\psi^{-3} g\right)^{\prime}\right] d t}\left[|c|+\int_{T}^{t}\left(\lambda_{1}^{* *} \xi_{1}+\lambda_{2}^{* *} \xi_{2}+D_{1}^{* *}+\right.\right.
\end{aligned}
$$

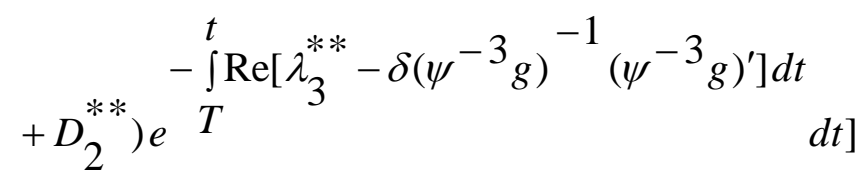

4- - النتائج الأساسية مبرهنة : في المعادلة (1) إذا كان

$$
\begin{array}{ll}
p_{i}: \Delta=[a, \infty] \rightarrow c, \quad h: \Delta \times C^{n} \rightarrow c & -1 \\
\left|h\left(t, y_{s} y^{\prime}, y^{n}\right)\right| \leq L^{*}\left[|y|+\left|y^{\prime}\right|+\left|y^{n}\right|\right]^{1+\beta} & \\
L^{*}: \Delta \rightarrow[0, \infty], \beta \geq 0, g: \Delta \rightarrow R & \\
\lim _{t \rightarrow \infty} \Psi^{-2} \Psi^{\prime}=0(1), \lim _{t \rightarrow \infty} W_{i}(t)=0(1) & -2
\end{array}
$$

وإذا كان

$$
\begin{aligned}
& \int_{T}^{t} R e \lambda d t \rightarrow \infty \text { as } t \rightarrow \infty \quad-\mathrm{A} \\
& \int_{T}^{t} \operatorname{Re} \lambda d t \int_{T}^{t} \lambda e^{-\int_{T}^{t} R e \lambda d t} d t=0(1), t \rightarrow \infty \quad-B \\
& \text { فان هذا يؤدي إلى أن الحل الصفري للمعادلة (1) مركزاً للجاذبية }
\end{aligned}
$$

وإذا كان

$\int_{T}^{t} \operatorname{Re} \lambda \mathrm{dt} \rightarrow \infty$ as $\mathrm{t} \rightarrow \infty-\mathrm{C}$

فان هذا يؤدي إلى أن الحل الصفري للمعادلة (1) لا تمثل مركزاً للجاذبية

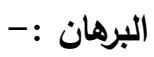

بتطبيق التحويلات (2) , (4) , (8) , (10) , (12) على المعادلة (1) نحصل على النظام المساعد $H_{1}^{\prime}=\left[\lambda_{1}-\delta\left(\psi^{-3} g\right)^{-1}\left(\psi^{-3} g\right)^{\prime}\right] H_{1}+\lambda_{2} \xi_{2}+\lambda_{3} \xi_{3}+D_{1}+D_{2}$

$H_{2}^{\prime}=\lambda_{1}^{*} \xi_{1}+\left[\lambda_{2}^{*}-\delta\left(\psi^{-3} g\right)^{-1}\left(\psi^{-3} g\right)^{\prime}\right] H_{2}+\lambda_{3}^{*} \xi_{3}+D_{1}^{*}+D_{2}^{*}$

$H_{3}^{\prime}=\lambda_{1}^{* *} \xi_{1}+\lambda_{2}^{* *} \xi_{2}+\left[\lambda_{3}^{* *}-\delta\left(\psi^{-3} g\right)^{-1}\left(\psi^{-3} g\right)^{\prime}\right] H_{3}+D_{1}^{* *}+D_{2}^{* *}$

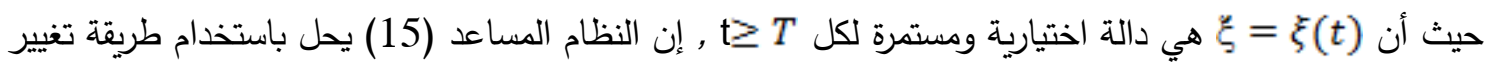

[5] الثوابت

$$
\begin{gathered}
\left|H_{1}\right| \leq e^{\int^{t} \operatorname{Re}\left[\lambda_{1}-\delta\left(\psi^{-3} g\right)^{-1}\left(\psi^{-3} g\right)^{\prime}\right] d t}\left[|c|+\int_{T}^{t}\left(\lambda_{2} \xi_{2}+\lambda_{3} \xi_{3}+D_{1}+\right.\right. \\
\left.\left.+D_{2}\right) e^{-\int_{T}^{t} \operatorname{Re}\left[\lambda_{1}-\delta\left(\psi^{-3} g\right)^{-1}\left(\psi^{-3} g\right)^{\prime}\right] d t} d t\right]
\end{gathered}
$$




$$
\begin{aligned}
& \left|H_{2}\right| \leq e^{\int^{t} \operatorname{Re}\left[\lambda_{2}^{*}-\delta\left(\psi^{-3} g\right)^{-1}\left(\psi^{-3} g\right)^{\prime}\right] d t}\left[|c|+\int_{T}^{t}\left(\lambda_{1}^{*} \xi_{1}+\lambda_{3}^{*} \xi_{3}+D_{1}^{*}+\right.\right. \\
& \left.+D_{2}^{*}\right) e^{T} \\
& -\int_{T}^{t} \operatorname{Re}\left[\lambda_{2}^{*}-\delta\left(\psi^{-3} g\right){ }^{-1}\left(\psi^{-3} g\right)^{\prime}\right] d t \\
& \int^{t} \operatorname{Re}\left[\lambda_{3}^{* *}-\delta\left(\psi^{-3} g\right)^{-1}\left(\psi^{-3} g\right)^{\prime}\right] d t \\
& \left|H_{3}\right| \leq e^{T}\left[|c|+\int_{T}^{t}\left(\lambda_{1}^{* *} \xi_{1}+\lambda_{2}^{* *} \xi_{2}+D_{1}^{* *}+\right.\right. \\
& \left.+D_{2}^{* *}\right) e^{T} \\
& -\int_{T}^{t} \operatorname{Re}\left[\lambda_{3}^{* *}-\delta\left(\psi^{-3} g\right)-1\left(\psi^{-3} g\right)^{\prime}\right] d t
\end{aligned}
$$
1) $e^{\int^{T} \operatorname{Re}\left[\lambda_{1}-\delta\left(\psi^{-3} g\right)^{-1}\left(\psi^{-3} g\right)^{\prime}\right] d t}=0$
$\int_{T}^{t} \operatorname{Re}\left[\lambda_{2}^{*}-\delta\left(\psi^{-3} g\right){ }^{-1}\left(\psi^{-3} g\right)^{\prime}\right] d t$
2) $e^{T} \quad=0$
$\int_{T}^{t} \operatorname{Re}\left[\lambda_{3}^{* *}-\delta\left(\psi^{-3} g\right){ }^{-1}\left(\psi^{-3} g\right)^{\prime}\right] d t$
3) $e^{T}$
$=0$

فان الحل الصفري للمعادلة (1) مركز جاذبية

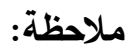

نظرا لصعوبة الحسابات الرياضية في هذه الحالة الحرجة فقد تم التعامل في هذا البحث مع معادلة تفاضلية من

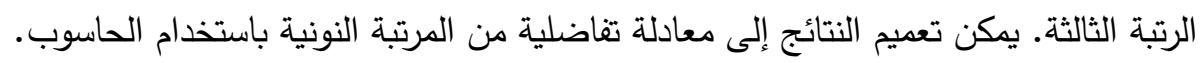




\section{المصادر}

[1] Cesari L., "Asymptotic behavior and stability problems in ordinary differential equation", 1963.

[2] Gantermager V.R., "Lectures in the analysis mechanics", Nauka Publications, Moscow, 1971.

[3] kostin A.V., I.E. Vitricenko, "Generalization of lyapunov's stability theorem in the case of one zero characteristic exponent for non-autonomous system", soviet math. Dokl, vol 25, no.3, 1982.

[4] Malkin I.G., Theory of stability of motion", Moscow, 1966.

[5] Sgolts L.E., "differential equation and calculus of variation", India, 1977.

[6] Tenenbaum M., Pollard H., "Ordinary differential equation", 1964.

[7] Thanoon T.Y., "Center of gravity of non autonomous non-linear differential system in some critical cases " Ph.D. thesis, Odessa, USSR, 1990.

[8] Thanoon T.Y., "stable solution nonautonomous semi-linear differential equation 2-nd order with slowly changeable coefficients", j.edh.sci,vol(28),1988.

[9] Thanoon T.Y., "On gravity curve of solution for non automous semi-linear differential equation 2 nd order with parameter", J. edu. Sci., Vol 29, P.P 124129, 1998.

[10] Thanoon T.Y., Al katib A.Z.,"the finding conditions of stable trivial solution for certian differential equation in the semi-linear-cases", J.Edu. Sci., vol 37 , p.p 88-96, 1999.

[11] Thanoon T.Y., Etewi R.J., "On the center of gravity for Quasi-linear differential equation of order $\mathrm{N}$ in the critical case", J.Edu. Sci., vol 12, no. 1, p.p 82-99, 2001. 